\title{
ON QUEUES WITH IMPATIENCE
}

\section{ROBERT E. STANFORD, ${ }^{*}$ The University of Alabama at Birmingham}

Volume 16 (1984) of Advances in Applied Probability contains a paper entitled 'Singleserver queues with impatient customers', by Baccelli, Boyer and Hebuterne (pp. 887-905), referred to below as B. The authors of this paper were unaware of some research of a similar nature previously published in the literature, the most recent being 'Reneging phenomena in single channel queues', by R. E. Stanford, which appeared in Vol. 4 (May 1979) of Mathematics of Operations Research, pp. 162-178. This paper is subsequently designated S. In communications with Professor Baccelli, this author has learned of more results of research on impatience, or reneging, in general queueing systems, specifically the papers 'General customer impatience in the queue GI/G/1', Journal of Applied Probability, Vol. 2 (1965), pp. 186-205, by D. J. Daley, and 'Existence of a limit distribution in queueing systems with bounded sojourn time', Theory of Probability and Its Applications, Vol. 10 (1965), pp. 515-522, by L. G. Afanas'eva (translated from Russian).

The purpose of this note is to provide a bibliographical update on the subject of impatience in general queueing systems, and to outline some of the similarities and differences in the results from the existing papers on the subject. All of the papers mentioned here are concerned with $G I / G / 1$ queues in which a customer drops out of the queue if his waiting time exceeds his patience time, which is a random variable.

Many studies of reneging phenomena have been carried out for queueing systems with specific (eg. exponential) interarrival, service, or reneging distributions. The combined list of references from S, B, and Daley (1965) provide an extensive bibliography of this research. Investigations of the $G I / G / 1$ system with a general reneging distribution appear to have begun with a model proposed by Kovalenko (1961). Necessary and sufficient conditions for existence of a limiting distribution for the waiting time in a $G I / G / 1$ queue in which the customer's wait is bounded by a random variable are given in Afanas'eva (1965). Daley (1965) also gives conditions for the existence of the limiting waiting time distribution, and derives an integral equation for the distribution. The integral equation is solved for certain special cases previously examined in the literature.

The two more recent papers $B$ and $S$ also contain sufficient conditions for a unique stationary distribution for the offered waiting time of arriving customers, and both contain new material concerning various characteristics of the $G I / G / 1$ system with reneging. In some cases, the results from the two papers are identical except for notation. A partial list of the expressions in $B$ which have a counterpart in $S$ is given below, by description and expression number, followed by the corresponding relation number from $S$ : definition of the offered waiting time-(2.1) in B, (7) in S; the limiting distribution of virtual waiting time-(3.6) in B, (20) in S; equations involving the probability of rejection (reneging)-(3.7) and (3.8) in B, (27) and (28) in S; a Pollaczek-Kintchine formula analog-remark \#1 following (3.11) in $\mathrm{B},(23)$ in $\mathrm{S}$; a relationship between the limiting distribution for virtual and actual waiting time-(3.11) in B, equivalent to (21) in S; an integral equation for the distribution of waiting times-(2.3) in B, (43) in S. The approaches of B and S do differ in the manner of derivation of many of the results that they have in common. The most significant difference concerns the determination of sufficient conditions for a unique stationary distribution for the offered waiting times of customers arriving at the queueing facility. The conditions required by $B$ are expressed in Lemma 2, section (2.3) of that paper; the more restrictive conditions required by $\mathrm{S}$ are expressed in Theorem 9 of that paper. We should also point out that the second part of

Received 22 December 1989; revision received 4 June 1990.

* Postal address: School of Business/Graduate School of Management, Department of Economics, The University of Alabama at Birmingham, AL 35294, USA. 
relation (26) in S does not follow directly from Wald's equation, as stated, without expression of the length of the first busy period, $B_{1}$, in terms of the service times of customers who do not renege. However, if we define $\bar{Y}_{j} \equiv$ service time of the $j$ th customer to reach the server, then for $j \geqq 1$, the variables $\bar{Y}_{j}$ are independent and identically distributed with the originally defined variables $Y_{j} \equiv$ potential service time of the $j$ th arrival to the system, and

$$
B_{1}=\sum_{j=1}^{K_{1}} \bar{Y}_{j},
$$

where $K_{1}=$ number of customers reaching the server during the first busy cycle. Since $K_{1}$ is a stopping time for the sequence of $\bar{Y}_{j}^{\prime} \mathrm{s}$, Wald's equation yields the second expression in relation (26) of $S$. In contrast, the same result follows from an extended Wald's formula given on p. 894 of $B$.

Most of the results presented in B concern the limiting distributions of virtual and actual waiting times of customers. Generalizations of other important queueing formulae to situations involving reneging are contained in $S$, including expressions equivalent to Little's formula and results concerning the distributions of the numbers of customers waiting in the system. Also, S contains a review of many well-known results for queues with specialized distributions for arrival, service and reneging, and derives these results as special cases of the more general assumptions of S. A few of these results duplicate material in Daley (1965).

In conclusion, it is clear that much work has been done on the subject of $G I / G / 1$ queueing systems with impatience, or reneging, and some duplication of results has occurred over the past couple of decades because researchers are not aware of existing research. Factors which appear to have contributed to these circumstances are (i) inconsistent terminology used to identify and classify queueing systems (e.g., 'reneging' vs. 'impatience'), a condition which may result in well-intentioned but ineffective reviews of existing literature, and (ii) an understandable absence of communication between researchers residing in different countries and using different publication outlets. It is hoped that this note will help correct the situation existing in this particular area of queueing research activity.

\section{References}

AfANAS'EVA, L. G. (1965) Existence of a limit distribution in queueing systems with bounded sojourn time. Theory Prob. Appl. 10, 515-522.

BACCELl, F., BOYER, P. AND Hebuterne, G. (1984) Single-server queues with impatient customers. Adv. Appl. Prob. 16, 887-905.

DALEY, D. J. (1965) General customer impatience in the queue GI/G/1. J. Appl. Prob. 2, 186-205.

Kovalenko, I. N. (1961) Some queueing problems with restrictions. Theory Prob. Appl. 6, 204-208.

StANFORD, R. E. (1979) Reneging phenomena in single channel queues. Math. Operat. Res. 4, 162-178. 